\title{
Efficient microwave-to-optical conversion using Rydberg atoms
}

\author{
Thibault Vogt, ${ }^{1,2}$ Christian Gross, ${ }^{1}$ Jingshan Han, ${ }^{1}$ Sambit B. Pal, ${ }^{1}$ Mark Lam, ${ }^{1}$ Martin Kiffner, ${ }^{1,3}$ and Wenhui Li ${ }^{1,4}$ \\ ${ }^{1}$ Centre for Quantum Technologies, National University of Singapore, 3 Science Drive 2, Singapore 117543 \\ ${ }^{2}$ MajuLab, CNRS-UCA-SU-NUS-NTU International Joint Research Unit UMI 3654, Singapore 117543 \\ ${ }^{3}$ Clarendon Laboratory, University of Oxford, Parks Road, Oxford OX1 3PU, United Kingdom \\ ${ }^{4}$ Department of Physics, National University of Singapore, 117542, Singapore
}

(Received 11 December 2018; published 19 February 2019)

\begin{abstract}
We demonstrate microwave-to-optical conversion using six-wave mixing in cold ${ }^{87} \mathrm{Rb}$ atoms where the microwave field couples to two Rydberg states and propagates collinearly with the converted optical field. Our experiment is performed with a free-space microwave field, and we achieve a conversion efficiency of about $5 \%$ for the microwave photons entering the conversion medium. In addition, we theoretically investigate all-resonant six-wave mixing and outline a realistic experimental scheme for reaching an efficiency close to $70 \%$.
\end{abstract}

DOI: 10.1103/PhysRevA.99.023832

\section{INTRODUCTION}

Rydberg atoms feature a quasicontinuum of narrow and strong dipole transitions coupling to microwave and terahertz (THz) radiation [1]. Moreover, they can be easily excited and manipulated with laser light. These two properties endow Rydberg atoms with tremendous potential for applications combining optical light with microwaves or THz waves in precision spectroscopy, quantum sensing, and information processing. For example, real-time $\mathrm{THz}$ field imaging has been achieved by $\mathrm{THz}$-induced optical fluorescence in Rydberg atoms [2]. Sensitive detection of free-space microwave fields based on dressed electromagnetically induced transparency involving a Rydberg state (EIT) is being actively pursued $[3,4]$. Rydberg EIT can also be used to transfer digital information encoded in a microwave field onto an optical carrier, with applications in radio-over-fiber technologies [5,6].

Recently, microwave-to-optical conversion has been demonstrated employing frequency mixing via Rydberg states [7]. The good coherence of the underlying process, characterized in Ref. [7], makes it a solid candidate for the transfer of quantum information between superconducting and photonic qubits. Among the different technologies being developed for this application [8-13], the highest microwave-optical photon conversion efficiency obtained so far is nearly $50 \%$ but with a relatively narrow bandwidth of $\sim 12 \mathrm{kHz}$ [12]. While the bandwidth of the converter using frequency mixing in Rydberg atoms demonstrated in [7] was as large as $4 \mathrm{MHz}$, the photon conversion efficiency was limited to $0.3 \%$. It thus remains to be shown that much higher efficiencies can be achieved with this approach.

In this paper, we demonstrate six-wave mixing in a cold rubidium $(\mathrm{Rb})$ gas where all the waves are near resonant with atomic transitions and propagate along a single axis. We show that the response of our converter is linear for a wide range of microwave input powers. Most importantly, the chosen configuration, with collinear propagation of the waves, enables us to enhance the conversion efficiency by a factor of 17 compared to that in [7]. Moreover, we theoretically analyze all-resonant collinear six-wave mixing within the framework of Maxwell-Bloch equations and identify conditions for achieving near-unit efficiencies.

The paper is organized as follows. We describe the experimental setup in Sec. II and present our experimental results in Sec. III. In Sec. IV, we show that our data are in good agreement with a numerical simulation based on Maxwell-Bloch equations. Finally, in Sec. V we derive an approximate, analytical description of all-resonant six-wave mixing and propose an optimized configuration which reaches an efficiency of nearly $70 \%$.

\section{EXPERIMENTAL SETUP}

A cloud of cold polarized ${ }^{87} \mathrm{Rb}$ atoms is illuminated by four auxiliary electromagnetic fields as well as the microwave field $M$ to be converted, as shown in Fig. 1(a). By nonlinear frequency mixing of the six waves in the atomic medium, the field $\mathrm{M}$ is converted into the optical field $\mathrm{L}$. The chosen configuration of energy levels is displayed in Fig. 1(b). The six waves P, C, A, M, R, and L are near resonant with the atomic transitions shown in the figure, where $|1\rangle \equiv\left|5 S_{1 / 2}, F=2, m_{F}=2\right\rangle,|2\rangle \equiv\left|5 P_{3 / 2}, F=3, m_{F}=3\right\rangle$, $|3\rangle \equiv\left|30 D_{3 / 2}, m_{J}=1 / 2\right\rangle,|4\rangle \equiv\left|31 P_{3 / 2}, m_{J}=-1 / 2\right\rangle,|5\rangle \equiv$ $\left|30 D_{5 / 2}, m_{J}=1 / 2\right\rangle$, and $|6\rangle \equiv\left|5 P_{3 / 2}, F=2, m_{F}=1\right\rangle[14]$. In the absence of the microwave field $M$, the system is in the configuration of microwave dressed Rydberg EIT formed by the two optical waves $\mathrm{P}$ and $\mathrm{C}$, and the auxiliary microwave field $\mathrm{A}$. Once the $\mathrm{M}$ and $\mathrm{R}$ fields are added, the coherence induced between the ground state $|1\rangle$ and the intermediate state $|6\rangle$ triggers the generation of the converted optical field $\mathrm{L}$.

In our setup, the fields $\mathrm{P}, \mathrm{M}$, and $\mathrm{A}$ are copropagating along the quantization axis $z$ and counterpropagating with the fields $\mathrm{C}$ and $\mathrm{R}$. The frequency differences between transitions $|1\rangle \leftrightarrow|2\rangle$ vs $|1\rangle \leftrightarrow|6\rangle,|2\rangle \leftrightarrow|3\rangle$ vs $|6\rangle \leftrightarrow|5\rangle$, and $|4\rangle \leftrightarrow$ $|3\rangle$ vs $|4\rangle \leftrightarrow|5\rangle$ are $0.27,0.73$, and $0.45 \mathrm{GHz}$, respectively, including the Zeeman shifts due to the $6.1-\mathrm{G}$ bias magnetic field applied along the $z$ axis $[15,16]$. These differences are 


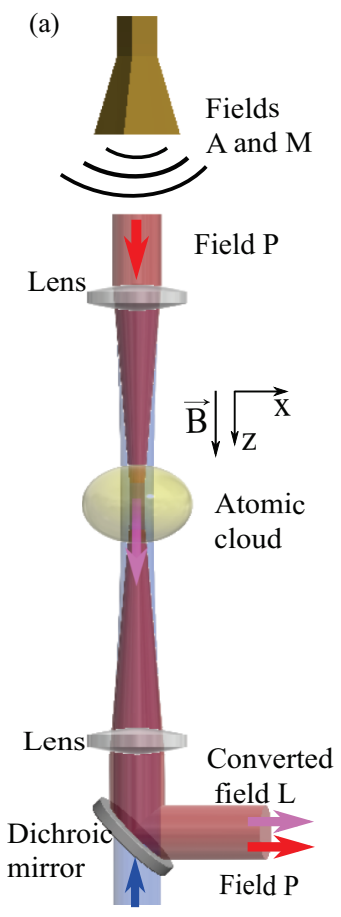

Fields $\mathrm{C}$ and $\mathrm{R}$ (b)

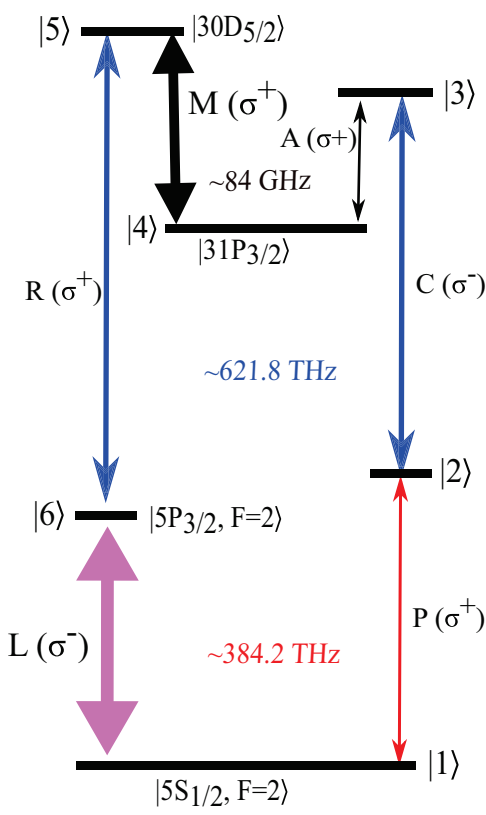

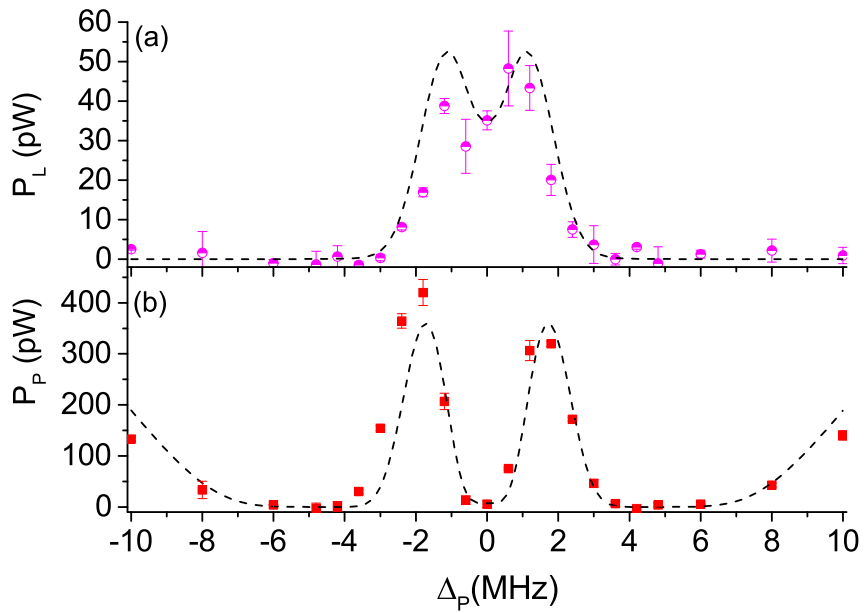

FIG. 2. Spectra of the generated light power $P_{L}$ (a) and of the transmitted $\mathrm{P}$ field power $P_{P}$ (b). The dashed lines are simulated results obtained using Maxwell-Bloch equations (see text).

$\left|d_{56}\right|=0.0138$. The latter four dipole matrix elements are obtained by numerical integration using the Numerov algorithm [17].

\section{EXPERIMENTAL RESULTS}

Typical spectra of the measured powers $P_{L}$ and $P_{P}$ of the $\mathrm{L}$ and $\mathrm{P}$ fields vs $\Delta_{P}$ are shown in Fig. 2. The peak Rabi frequencies of the incoming fields are $\Omega_{P 0} / 2 \pi=1.0 \mathrm{MHz}$, $\Omega_{C 0} / 2 \pi=9.5 \mathrm{MHz}, \quad \Omega_{R 0} / 2 \pi=6.3 \mathrm{MHz}, \quad \Omega_{A 0} / 2 \pi=$ $2.9 \mathrm{MHz}$, and $\Omega_{M 0} / 2 \pi=1.4 \mathrm{MHz}$. The data obtained for each $\Delta_{P}$ are averages of the signals recorded in steady-state condition over $10 \mu$ s after the application of the fields. The conversion is most efficient at around $\Delta_{P}=0$ [Fig. 2(a)], which is consistent with the nonlinearity responsible for the frequency mixing being maximum close to resonance. The spectra of the $\mathrm{L}$ and $\mathrm{P}$ fields in Fig. 2 are approximately symmetric. The double-peak shape of the spectrum of $P_{P}$ occurs mostly due to the large A field and is likely an effect of microwave dressed EIT on $|1\rangle \rightarrow|2\rangle \rightarrow|3\rangle \rightarrow|4\rangle$ [18]. We attribute the small reduction of $P_{L}$ at the center of the spectrum in Fig. 2(a) to the strong absorption of the $\mathrm{P}$ field around $\Delta_{P}=0$, which tends to reduce the effective volume of the medium where the conversion occurs. We have observed a disappearance of the dip in the $\mathrm{L}$ field spectrum at lower microwave A power, consistent with this interpretation. Indeed, when the microwave A power is reduced, the two EIT peaks in the spectrum of the $\mathrm{P}$ field tend to overlap, which leads to less attenuation of $P_{P}$ at $\Delta_{P}=0$.

The behavior of the measured power $P_{L}$ for $\Delta_{P}=0$ is approximately linear as a function of the input intensity $I_{M}$ of field M [see Fig. 3(a)]. The expected signal due to the conversion of blackbody radiation is several orders of magnitude smaller than the technical noise due to the detectors and hence has a negligible effect on the data of Fig. 3(a). Given $P_{L} \approx$ $\alpha I_{M}$, a linear fit to the data yields the slope $\alpha=0.45 \mathrm{~mm}^{2}$. The photon conversion efficiency of the process is calculated 

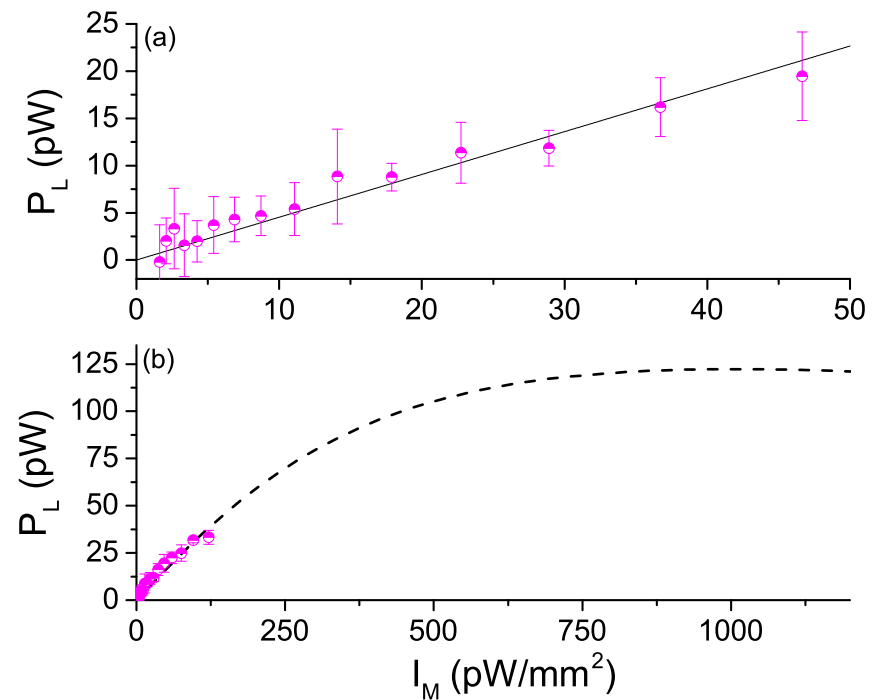

FIG. 3. The linearity of the converter. (a) The power of the generated light is plotted vs $I_{M}$ in the range of $0-50 \mathrm{pW} / \mathrm{mm}^{2}$. The solid line is the result of a linear fit to the data. (b) The power of the generated light is plotted vs $I_{M}$ in the range of $0-1200 \mathrm{pW} / \mathrm{mm}^{2}$. The dashed line is the numerically simulated result (see text).

as

$$
\eta \approx \frac{P_{L} / v_{L}}{I_{M} S_{M} / v_{M}}
$$

where $S_{M}=\pi w_{P}^{2}$ and $v_{L} \approx 384.228 \mathrm{THz}$, yielding $\eta=0.051$ for the data shown in Fig. 3(a). For this calculation, we only consider the microwave photons incident on the conversion medium, whose transverse size is determined by the probe beam waist $w_{P}$. This conversion efficiency is 17 times larger than that reported in Ref. [7] using a perpendicular configuration, where the microwave fields propagated at right angles to the optical fields. The physical reason for this enhancement is that the conversion occurs over a much longer distance in the collinear configuration $\left(\sim 2 w_{z}\right)$ than in the perpendicular geometry $\left(\sim 2 w_{P}\right)$. Note that the change of geometry implies a change of polarization of the microwave fields. As a consequence, $\left|d_{54}\right|$ has been reduced by a factor of $\sqrt{2}$ because the microwave field $\mathrm{M}$ drives a different atomic transition. Furthermore, we use a larger A field, which can be shown to increase the linear range of the converter but tends to reduce the efficiency. Because of these two reasons, the measured efficiency is lower than that predicted in Ref. [7].

\section{NUMERICAL SIMULATION}

Next, we numerically simulate the interaction of the laser and microwave fields with the atomic ensemble within the framework of coupled Maxwell-Bloch equations. We restrict the problem to the calculation of one-dimensional solutions for the fields in the slowly varying envelope approximation, and in steady state. With these assumptions, the field amplitudes satisfy the following differential equations:

$$
\partial_{z} \Omega_{X}=i \frac{n_{\mathrm{at}}\left|d_{j i}\right|^{2} v_{X}}{h \epsilon_{0} c} \varrho_{j i}
$$

In this set of equations, the complex amplitudes $\mathcal{E}_{X}$ of each field $X(X \in\{P, C, A, M, R, L\})$ have been rescaled using the definition of the Rabi frequency $\Omega_{X}=2 \mathcal{E}_{X} d_{j i} / \hbar$, where $\hbar$ is the reduced Planck constant and $d_{j i}$ is the dipole matrix element of the transition $|i\rangle \leftrightarrow|j\rangle$ driven by field $\mathrm{X}$. Moreover, $\varrho_{j i}$ is the corresponding steady-state atomic coherence, written in a suitable rotating frame removing fast temporal and spatial oscillations, $v_{X}$ is the frequency of field $\mathrm{X}, n_{a t}$ is the atomic density, $\epsilon_{0}$ is the free-space permittivity, and $c$ is the speed of light in vacuum. The atomic coherences are given by the steady-state solution $\varrho$ of the following Markovian master equation:

$$
\partial_{t} \rho=-\frac{i}{\hbar}[H, \rho]+\mathcal{L}_{\Gamma} \rho+\mathcal{L}_{\mathrm{deph}} \rho
$$

where

$$
H=-\frac{\hbar}{2}\left(\Delta_{P}|2\rangle\left\langle 2\left|+\sum_{X} \Omega_{X}\right| j\right\rangle\langle i|+\text { H.c. }\right)
$$

is the interaction Hamiltonian between a single atom and the fields and $\rho(t)$ is the (time-dependent) density matrix of the atom. The Lindblad term $\mathcal{L}_{\Gamma} \rho$ accounts for spontaneous emission from the intermediate states $|2\rangle$ and $|6\rangle$ with rate $\Gamma=2 \pi \times 6.067 \mathrm{MHz}$ and from the Rydberg states. The term $\mathcal{L}_{\operatorname{deph}} \rho$ accounts for additional dephasing mechanisms and may be written as

$$
\mathcal{L}_{\text {deph }} \rho=\sum_{j \in\{3,4,5\}} \gamma_{j}\left(2 P_{j} \rho P_{j}-P_{j} \rho-\rho P_{j}\right),
$$

where $P_{j}$ is the projection operator on state $|j\rangle$, and $\gamma_{j}$ is the dephasing rate associated with state $|j\rangle$. These rates include the effects of atomic collisions, dipole-dipole interactions between Rydberg atoms, and finite laser linewidths. The dephasing rates $\gamma_{1}, \gamma_{2}$, and $\gamma_{6}$ are neglected since they are much smaller than $\Gamma$.

For solving Eq. (2), the input Gaussian fields are assumed to be collimated, with transverse $1 / e$ radii given by the waist of the beams in absence of the atoms. The output intensity profiles obtained from these solutions are integrated radially to calculate the total output powers of the $\mathrm{P}$ and $\mathrm{L}$ fields. In deriving Eq. (2), we have neglected transverse terms in Maxwell equations that account for diffraction and lensing effects. This approximation yields relatively accurate results for our experimental parameters. Note that including these transverse terms would likely account for the slight asymmetry of the spectrum of $P_{P}$ observed in Fig. 2(b) [7,20].

The results of our numerical simulation are shown in Figs. 2(a), 2(b), and 3(b). They are calculated for $\gamma_{3}=2 \pi \times$ $50 \mathrm{kHz}, \gamma_{4}=2 \pi \times 300 \mathrm{kHz}$, and $\gamma_{5}=2 \pi \times 400 \mathrm{kHz}$, while the other input parameters are obtained from experimental calibrations. The good agreement with the data further asserts the validity of the model of Refs. [7,19] and allows us to extrapolate the conversion process to larger input microwave intensities, as shown in Fig. 3(b). While $P_{L}$ is linear at small $I_{M}$, it starts to saturate when $\Omega_{M 0}$ becomes of the order of $\Omega_{A 0}$. 


\section{ANALYTICAL INSIGHTS AND OPTIMIZED CONFIGURATION}

To present a clear pathway to improving the efficiency, we will take a closer look at Eq. (3) in the case of allresonant fields. We obtain the analytical steady-state solution of Eq. (3) as an expansion of $\varrho$ up to third order in the weak fields $\Omega_{P}, \Omega_{M}, \Omega_{L}$ and small dephasing rates $\gamma_{j}$, as shown in the Appendix. In keeping only the dominant terms, the coherences $\varrho_{21}, \varrho_{61}$, and $\varrho_{54}$ take the following simple form:

$$
\begin{gathered}
\varrho_{21}=i \frac{\Omega_{P}}{\Gamma}, \\
\varrho_{61}=i \frac{\Omega_{P} \Omega_{C} \Omega_{M}}{\Gamma \Omega_{A} \Omega_{R}}, \\
\varrho_{54}=-i \frac{\Omega_{R} \Omega_{L} \Omega_{A} \Omega_{C}^{*} \Omega_{P}^{*}-\left|\Omega_{C}\right|^{2}\left|\Omega_{P}\right|^{2} \Omega_{M}}{\Gamma\left|\Omega_{A}\right|^{2}\left|\Omega_{R}\right|^{2}},
\end{gathered}
$$

when the conditions $\Gamma\left|\Omega_{A}\right|^{2} /\left|\Omega_{C}\right|^{2} \gg \gamma_{4},\left|\Omega_{R}\right|^{2} / \Gamma \gg \gamma_{5}$, and $\left|\Omega_{C} \Omega_{R} \Omega_{M} \Omega_{P}\right| /\left|\Gamma \Omega_{A} \Omega_{L}\right| \gg \gamma_{5}$ are fulfilled.

Equations (6)-(8) do not depend on the dephasing rates. Therefore the conditions in the latter inequalities ensure that our system is robust against interaction-induced dephasing, and realizing them experimentally will lead to high efficiencies. In this regime, and taking the large Rabi frequencies $\Omega_{A}, \Omega_{C}$, and $\Omega_{R}$ as constants, we find from the system of Eqs. (6)-(8) and Eq. (2) that $\Omega_{L}$ satisfies the following differential equation:

$$
\partial_{v}^{2} \Omega_{L}+a_{2}(1-v) \partial_{v} \Omega_{L}+a_{1}^{2} \Omega_{L}=0,
$$

where $v$ is defined as $v(z)=1-e^{-u(z) / 2}$, with

$$
u(z)=\int_{-\infty}^{z} d z^{\prime} 2 n_{a t}\left|d_{21}\right|^{2} v_{P} /\left(h \epsilon_{0} c \Gamma\right)
$$

being the optical depth vs $z$ for the P field. Taking $v_{P} \approx v_{L}$, we have $a_{1}=c_{1}\left|d_{61} / d_{21}\right|^{2}$ and $a_{2}=c_{1}^{2}\left|d_{61} / d_{21}\right|^{2}$, where

$$
c_{1}=\left|\frac{d_{54}}{d_{61}}\right| \sqrt{\frac{v_{M}}{v_{L}}} \frac{\Omega_{C} \Omega_{P}(z=-\infty)}{\Omega_{R} \Omega_{A}} .
$$

The analytical solution to Eq. (9) can be expressed in terms of a hypergeometric function and a Hermite polynomial. Equation (9) is very similar to that of a damped harmonic oscillator, with the damping term linearly depending on $v$, as $a_{2}(1-v)$, where $0 \leqslant v<1$. By analogy, when $a_{2} \ll a_{1}$ the solution takes the simple form $\Omega_{L}(v) \approx \Omega_{L 0} \sin \left(a_{1} v\right)$, where

$$
\Omega_{L 0}=\sqrt{\frac{v_{L}}{v_{M}}}\left|\frac{d_{61}}{d_{54}}\right| \Omega_{M}(z=-\infty)
$$

corresponds to $100 \%$ conversion efficiency. Therefore optimizing the efficiency implies to fulfill the two conditions $a_{1} v(+\infty)=\pi / 2$ and $a_{2} \ll a_{1}$. For a large enough atomic cloud, this can be achieved by decreasing $\left|d_{21}\right|$ and increasing $\left|d_{61}\right|$. Physically, the idea is to minimize the absorption of the $\mathrm{P}$ field while maximizing the converted $\mathrm{L}$ field.

Based on this analytical derivation, we select a more favorable configuration of energy levels where $\left|d_{21}\right|$ is reduced by a factor of $\sqrt{15}$, and $\left|d_{61}\right|$ increased by $\sqrt{6}$. The new configuration is such that $\left|1^{\prime}\right\rangle \equiv|1\rangle,\left|2^{\prime}\right\rangle \equiv\left|5 P_{3 / 2}, F=3, m_{F}=1\right\rangle$,

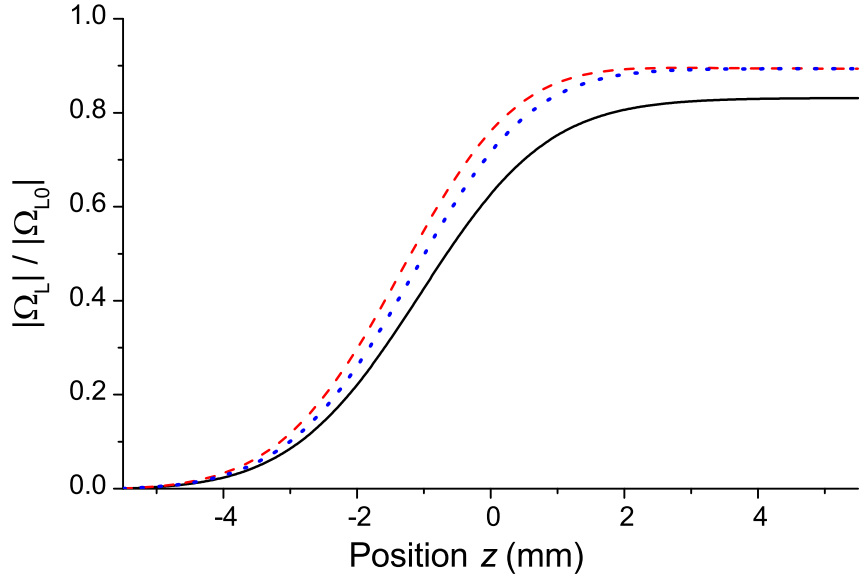

FIG. 4. Optimizing the converted field L. The dashed red line represents the analytical solution of the L field Rabi frequency $\Omega_{L}$ vs position $z$ inside a Gaussian-distributed atomic sample. The calculation is based on the result of Eq. (9), with $a_{1} v(+\infty)=\pi / 2$ (see text). The dotted blue line is a full numerical simulation of $\Omega_{L}$ vs $z$, without considering dephasing rates. The solid black line is the full numerical simulation of $\Omega_{L}$ vs $z$, including the dephasing rates $\gamma_{3}, \gamma_{4}$, and $\gamma_{5}$. For all three curves, $\Omega_{L}$ is rescaled with the Rabi frequency $\Omega_{L 0}$ corresponding to $100 \%$ efficiency.

$\left|3^{\prime}\right\rangle \equiv|3\rangle, \quad\left|4^{\prime}\right\rangle \equiv\left|31 P_{3 / 2}, m_{J}=3 / 2\right\rangle, \quad\left|5^{\prime}\right\rangle \equiv \mid 30 D_{5 / 2}, m_{J}=$ $5 / 2\rangle$, and $\left|6^{\prime}\right\rangle \equiv\left|5 P_{3 / 2}, F=3, m_{F}=3\right\rangle$, and we keep the fields as denominated in Fig. 1 but with modified polarizations and strengths. We consider extended input fields of Rabi frequencies $\Omega_{P 0} / 2 \pi=1.0 \mathrm{MHz}, \Omega_{C 0} / 2 \pi=13.5 \mathrm{MHz}$, $\Omega_{R 0} / 2 \pi=29 \mathrm{MHz}, \Omega_{A 0} / 2 \pi=9.2 \mathrm{MHz}$, and a Gaussiandistributed atomic cloud with $n_{a t, 0}=2 \times 10^{10} \mathrm{~cm}^{-3}$ and $w_{z}=3.7 \mathrm{~mm}$, such that $a_{1} v(+\infty) \approx \pi / 2$. Figure 4 compares the analytical solution of Eq. (9) to the numerical simulation of $\Omega_{L}$ given by Eqs. (2) and (3) without dephasing $\left(\mathcal{L}_{\text {deph }}=0\right)$ as a function of the position $z$ along the atomic cloud. The two curves are in good agreement, and the remaining discrepancy would vanish for $\Omega_{P 0} / 2 \pi<0.5 \mathrm{MHz}$. Moreover, the introduction of finite dephasing rates similar to those measured in our experiment [see Eq. (5)] only moderately affects the numerical simulation, as expected from our initial assumptions and shown in Fig. 4 as well. The efficiency $\eta$, deduced directly from these solutions, is of about $69 \%$ if the dephasing rates are taken into account, in comparison to about $80 \%$ without dephasing. This system would likely require a larger bias magnetic field and active optical pumping to compensate for the depumping effect of the $\sigma^{-}$-polarized $\mathrm{P}$ field, which drives an open transition. A possible approach to further improving $\eta$ is to rely on a higher-lying state $|2\rangle$ such that $\left|d_{21}\right|$ is significantly reduced.

An important feature of our scheme is its large conversion bandwidth. We have numerically simulated the spectrum of $P_{L}$ vs microwave field detuning for the parameters used in Fig. 4 and extracted a full width at half maximum of nearly $15 \mathrm{MHz}$, which is large and very promising for applications that require large conversion rates. This width is about 3 times wider than the one calculated with the parameters of Fig. 3. This increase is mostly due to the larger Rabi frequency $\Omega_{R}$ used in the improved configuration of Fig. 4. 


\section{CONCLUSION}

In conclusion, we have experimentally realized microwave-to-optical conversion using all-resonant, free-space, and collinear six-wave mixing via Rydberg states. At low microwave powers, the converter is linear and has a photon conversion efficiency of about $5 \%$. Moreover, we have derived an analytical solution to all-resonant six-wave mixing. This solution was used to identify a range of experimental parameters where the conversion process is approximately insensitive to interaction-induced dephasing and where high conversion efficiencies are reachable with cold atom clouds.

This conversion method using Rydberg atoms is promising for the sensitive detection of microwave or $\mathrm{THz}$ photons, since these photons could be very efficiently transferred into optical photons before photodetection. It is also promising for quantum state transfer. Converting microwave fields in a frequency range suitable for circuit quantum electrodynamics experiments should be possible by using higher $n$ states and/or different atomic species such as caesium.

To reach a high fidelity for quantum state transfer, one may consider stimulated Raman adiabatic passage, a technique which may be applicable to our system [21]. Another option that in theory achieves near-unit conversion efficiency is to tune two of the fields off-resonance to realize an effective twophoton transition, for example, fields $\mathrm{C}$ and $\mathrm{A}$ in our system $[19,22]$. Eventually, the realization of microwave-to-optical conversion at the single photon level for sensing or quantum applications will require a tightly focused or confined microwave field and integration of the system into a noise-free environment [23-25].

\section{ACKNOWLEDGMENTS}

The authors acknowledge support from the National Research Foundation, Prime Minister's Office, Singapore, and the Ministry of Education, Singapore, under the Research Centres of Excellence program. This work is supported by the Singapore Ministry of Education Academic Research Fund Tier 2 (Grant No. MOE2015-T2-1-085).

\section{APPENDIX}

Here we describe how to derive the analytical expressions of the steady-state coherences $\varrho_{j i}$ in Eqs. (6)-(8) using perturbation theory $[19,26]$. To this end, we assume that $\Gamma, \Omega_{A}$, $\Omega_{C}$, and $\Omega_{R}$ are much larger than $\Omega_{P}, \Omega_{M}, \Omega_{L}$, and the dephasing rates $\gamma_{j}(j \in\{3,4,5\})$, and assume that the steadystate density matrix $\varrho$ can be expanded in a perturbation series of the form

$$
\varrho=\sum_{k=0}^{\infty} \varrho^{(k)} .
$$

Moreover, we restrict the problem to $\Delta_{P}=0$ and neglect the decay rates of the Rydberg states, which are much smaller than all the other decay rates of interest in our experiment [27]. The master equation, Eq. (3), may be rewritten as

$$
\partial_{t} \rho=\mathcal{L}_{0} \rho+\mathcal{L}_{1} \rho+\mathcal{L}_{\Gamma} \rho+\mathcal{L}_{\mathrm{deph}} \rho
$$

where

$$
\mathcal{L}_{0} \rho=\frac{1}{2}\left[i\left(\Omega_{A}|3\rangle\left\langle 4\left|+\Omega_{C}\right| 3\right\rangle\left\langle 2\left|+\Omega_{R}\right| 5\right\rangle\langle 6|\right)+\text { H.c., } \rho\right]
$$

and

$$
\mathcal{L}_{1} \rho=\frac{1}{2}\left[i\left(\Omega_{L}|6\rangle\left\langle 1\left|+\Omega_{M}\right| 5\right\rangle\left\langle 4\left|+\Omega_{P}\right| 2\right\rangle\langle 1|\right)+\text { H.c., } \rho\right] .
$$

The zeroth-order solution in steady state, $\varrho^{(0)}$, is obtained after keeping only the zeroth-order terms in Eq. (A2), i.e., solving the equation

$$
\mathcal{L}_{0} \varrho^{(0)}+\mathcal{L}_{\Gamma} \varrho^{(0)}=0
$$

Inserting the expansion of Eq. (A1) into Eq. (A2) leads in steady state to a set of differential equations relating the $k$ th-order solution $\varrho^{(k)}$ to $\varrho^{(k-1)}$ as

$$
\mathcal{L}_{0} \varrho^{(k)}+\mathcal{L}_{\Gamma} \varrho^{(k)}+\mathcal{L}_{1} \varrho^{(k-1)}+\mathcal{L}_{\mathrm{deph}} \varrho^{(k-1)}=0 .
$$

Starting from the result of Eq. (A5), Eq. (A6) can be solved iteratively to yield all the orders $\varrho^{(k)}, k>0$ under the constraints $\operatorname{Tr}\left(\varrho^{(0)}\right)=1$ and $\operatorname{Tr}\left(\varrho^{(k)}\right)=0 \quad(k>0)$. The atomic coherences of interest are given below up to third order:

$$
\begin{gathered}
\varrho_{21}^{(0)}=\varrho_{61}^{(0)}=\varrho_{54}^{(0)}=\varrho_{43}^{(0)}=\varrho_{61}^{(1)}=\varrho_{54}^{(1)}=\varrho_{43}^{(1)}=0, \\
\varrho_{21}^{(1)}=i \frac{\Omega_{P}}{\Gamma}, \\
\varrho_{21}^{(2)}=i \frac{\Omega_{P}}{\Gamma}\left(C_{1}-C_{2}\right), \\
\varrho_{61}^{(2)}=i \frac{\Omega_{P} \Omega_{C} \Omega_{M}}{\Gamma \Omega_{A} \Omega_{R}}\left(1+C_{3}\right), \\
\varrho_{54}^{(2)}=-i \frac{\Omega_{L} \Omega_{C}^{*} \Omega_{P}^{*}}{\Gamma \Omega_{A}^{*} \Omega_{R}^{*}}, \\
\varrho_{61}^{(3)}=i \frac{\Omega_{P} \Omega_{C} \Omega_{M}}{\Gamma \Omega_{A} \Omega_{R}}\left(C_{1}-C_{2}-C_{4}-C_{3} C_{4}-C_{3} \frac{\left|\Omega_{P}^{2}\right|}{2 \Gamma \gamma_{5}}\right) \\
\varrho_{54}^{(3)}=0 \\
i \frac{\left|\Omega_{C}\right|^{2}\left|\Omega_{P}\right|^{2} \Omega_{M}}{\Gamma\left|\Omega_{A}\right|^{2}\left|\Omega_{R}\right|^{2}}+i \varrho_{54}^{(2)}\left(C_{1}^{*}-C_{2}-C_{4}\right), \\
\varrho_{43}^{(3)}=-i \frac{\Omega_{L}^{*} \Omega_{M} \Omega_{P} \Omega_{C}}{\Omega_{A}^{2} \Omega_{R} \Gamma},
\end{gathered}
$$

where we have omitted $\varrho_{21}^{(3)}$ since $\varrho_{21}$ is already nonzero at first order. The correction coefficients $C_{1}, C_{2}, C_{3}$, and $C_{4}$ are defined for comparison with the dominant terms as

$$
\begin{gathered}
C_{1}=\frac{\Omega_{L} \Omega_{M}^{*} \Omega_{C}^{*}}{\Omega_{P} \Omega_{A}^{*} \Omega_{R}^{*}}, \\
C_{2}=\frac{2 \gamma_{4}\left|\Omega_{c}\right|^{2}}{\Gamma\left|\Omega_{A}\right|^{2}}, \\
C_{3}=\frac{2 \gamma_{5} \Omega_{L} \Omega_{A} \Gamma}{\Omega_{C} \Omega_{R}^{*} \Omega_{M} \Omega_{P}}, \\
C_{4}=\frac{2 \gamma_{5} \Gamma}{\left|\Omega_{R}\right|^{2}} .
\end{gathered}
$$


These coefficients have to be significantly smaller than 1 to recover Eqs. (6)-(8). For the optimized configuration discussed in Sec. V and shown in Fig. 3, these coefficients take the maximum values of $C_{1, \max } \approx 0.18 \times 10^{-3}, C_{2, \max } \approx 0.21$, $C_{3, \max } \approx 0.28$, and $C_{4, \max } \approx 0.58 \times 10^{-2}$. Furthermore, we find that $C_{3} \ll C_{3, \max }$ on a large portion of the conversion medium.
[1] T. F. Gallagher, Rydberg Atoms (Cambridge University Press, Cambridge, 1994).

[2] C. G. Wade, N. Šibalić, N. R. de Melo, J. M. Kondo, C. S. Adams, and K. J. Weatherill, Real-time near-field terahertz imaging with atomic optical fluorescence, Nat. Photon. 11, 40 (2016).

[3] J. A. Sedlacek, A. Schwettmann, H. Kübler, R. Löw, T. Pfau, and J. P. Shaffer, Microwave electrometry with Rydberg atoms in a vapour cell using bright atomic resonances, Nat. Phys. 8, 819 (2012).

[4] M. T. Simons, J. A. Gordon, C. L. Holloway, D. A. Anderson, S. A. Miller, and G. Raithel, Using frequency detuning to improve the sensitivity of electric field measurements via electromagnetically induced transparency and Autler-Townes splitting in Rydberg atoms, Appl. Phys. Lett. 108, 174101 (2016).

[5] D. H. Meyer, K. C. Cox, F. K. Fatemi, and P. D. Kunz, Digital communication with Rydberg atoms and amplitude-modulated microwave fields, Appl. Phys. Lett. 112, 211108 (2018).

[6] A. Deb and N. Kjærgaard, Radio-over-fiber using an optical antenna based on Rydberg states of atoms, Appl. Phys. Lett. 112, 211106 (2018).

[7] J. Han, T. Vogt, C. Gross, D. Jaksch, M. Kiffner, and W. Li, Coherent Microwave-to-Optical Conversion via Six-Wave Mixing in Rydberg Atoms, Phys. Rev. Lett. 120, 093201 (2018).

[8] R. Hisatomi, A. Osada, Y. Tabuchi, T. Ishikawa, A. Noguchi, R. Yamazaki, K. Usami, and Y. Nakamura, Bidirectional conversion between microwave and light via ferromagnetic magnons, Phys. Rev. B 93, 174427 (2016).

[9] A. Rueda, F. Sedlmeir, M. C. Collodo, U. Vogl, B. Stiller, G. Schunk, D. V. Strekalov, C. Marquardt, J. M. Fink, O. Painter, G. Leuchs, and H. G. L. Schwefel, Efficient microwave to optical photon conversion: An electro-optical realization, Optica 3, 597 (2016).

[10] J. Bochmann, A. Vainsencher, D. D. Awschalom, and A. N. Cleland, Nanomechanical coupling between microwave and optical photons, Nat. Phys. 9, 712 (2013).

[11] T. Bagci, A. Simonsen, S. Schmid, L. G. Villanueva, E. Zeuthen, J. Appel, J. M. Taylor, A. Sorensen, K. Usami, A. Schliesser, and E. S. Polzik, Optical detection of radio waves through a nanomechanical transducer, Nature (London) 507, 81 (2014).

[12] A. P. Higginbotham, P. S. Burns, M. D. Urmey, R. W. Peterson, N. S. Kampel, B. M. Brubaker, G. Smith, K. W. Lehnert, and C. A. Regal, Harnessing electro-optic correlations in an efficient mechanical converter, Nat. Phys. 14, 1038 (2018).

[13] I. Lekavicius, D. A. Golter, T. Oo, and H. Wang, Transfer of Phase Information between Microwave and Optical Fields via an Electron Spin, Phys. Rev. Lett. 119, 063601 (2017).
[14] Note that the hyperfine structure of the considered Rydberg states is negligible.

[15] J. Ye, S. Swartz, P. Jungner, and J. L. Hall, Hyperfine structure and absolute frequency of the $87 \mathrm{Rb} 5$ p $3 / 2$ state, Opt. Lett. 21, 1280 (1996).

[16] M. Mack, F. Karlewski, H. Hattermann, S. Höckh, F. Jessen, D. Cano, and J. Fortágh, Measurement of absolute transition frequencies of $\mathrm{Rb} 87$ to $\mathrm{nS}$ and $\mathrm{nD}$ Rydberg states by means of electromagnetically induced transparency, Phys. Rev. A 83, 052515 (2011).

[17] M. L. Zimmerman, M. G. Littman, M. M. Kash, and D. Kleppner, Stark structure of the Rydberg states of alkali-metal atoms, Phys. Rev. A 20, 2251 (1979).

[18] M. Tanasittikosol, J. D. Pritchard, D. Maxwell, A. Gauguet, K. J. Weatherill, R. M. Potvliege, and C. S. Adams, Microwave dressing of Rydberg dark states, J. Phys. B 44, 184020 (2011).

[19] M. Kiffner, A. Feizpour, K. T. Kaczmarek, D. Jaksch, and J. Nunn, Two-way interconversion of millimeter-wave and optical fields in Rydberg gases, New J. Phys. 18, 093030 (2016).

[20] J. Han, T. Vogt, M. Manjappa, R. Guo, M. Kiffner, and W. $\mathrm{Li}$, Lensing effect of electromagnetically induced transparency involving a Rydberg state, Phys. Rev. A 92, 063824 (2015).

[21] B. T. Gard, K. Jacobs, R. McDermott, and M. Saffman, Microwave-to-optical frequency conversion using a cesium atom coupled to a superconducting resonator, Phys. Rev. A 96, 013833 (2017).

[22] T. Vogt, C. Gross, T. F. Gallagher, and W. Li, Microwaveassisted Rydberg electromagnetically induced transparency, Opt. Lett. 43, 1822 (2018).

[23] S. D. Hogan, J. A. Agner, F. Merkt, T. Thiele, S. Filipp, and A. Wallraff, Driving Rydberg-Rydberg Transitions from a Coplanar Microwave Waveguide, Phys. Rev. Lett. 108, 063004 (2012).

[24] C. Hermann-Avigliano, R. C. Teixeira, T. Nguyen, T. CantatMoltrecht, G. Nogues, I. Dotsenko, S. Gleyzes, J. Raimond, S. Haroche, and M. Brune, Long coherence times for Rydberg qubits on a superconducting atom chip, Phys. Rev. A 90, 040502 (2014).

[25] D. Cano, H. Hattermann, B. Kasch, C. Zimmermann, R. Kleiner, D. Koelle, and J. Fortágh, Experimental system for research on ultracold atomic gases near superconducting microstructures, Eur. Phys. J. D 63, 17 (2011).

[26] R. W. Boyd, M. S. Malcuit, D. J. Gauthier, and K. Rzaewski, Competition between amplified spontaneous emission and the four-wave-mixing process, Phys. Rev. A 35, 1648 (1987).

[27] D. B. Branden, T. Juhasz, T. Mahlokozera, C. Vesa, R. O. Wilson, M. Zheng, A. Kortyna, and D. A. Tate, Radiative lifetime measurements of rubidium Rydberg states, J. Phys. B 43, 015002 (2010). 